\title{
A Potential Method for the Nonuniformity Correction and Noise Removal of Infrared Thermal Image
}

\author{
Xiangyu Zeng, Jun Xu and Xiumin GaO* \\ University of Shanghai for Science and Technology, Shanghai 200093, China \\ (Received August 30, 2019; in final form March 3, 2020)
}

\begin{abstract}
Nonuniformity and noise are the two crucial factors that reduce the sharpness of infrared thermal images. It is essential to correct the nonuniformity and remove the noise of thermal images. A method for the nonuniformity correction and noise removal of the infrared thermal images that combines convolutional neural networks with a double-Gaussian filter was proposed. To demonstrate the advantages of this method, the values of roughness and nonuniformity of 300 infrared thermal images with different degrees of nonuniformity captured from various focal distances and fields of view were analyzed based by combined convolutional neural networks with a doubleGaussian filter. Furthermore, the results of that were compared in the form of a line chart with other commonly used algorithms. As a result, combined convolutional neural networks with a double-Gaussian filter was neither the best nor the worst one from the point of roughness and nouniformity while it was the best from a comprehensive performance of view. So, combined convolutional neural networks with a double-Gaussian filter may be a potential method for the nonuniformity correction and denoising of the infrared thermal image.
\end{abstract}

DOI: 10.12693/APhysPolA.137.1055

PACS/topics: correction of nonuniformity, denoising, convolutional neural network based on double-Gaussian filter (DGCNN), thermal image

\section{Introduction}

The noise and nonuniformity of infrared images are mainly caused by the different outputs of infrared detector array, and influenced by the ambient temperature of the infrared camera and the internal temperature of the detector [1]. Two kinds of methods were carried out by the previous researchers to remove the noise and nonuniformity of the infrared images. The first one is calibration-based correction, which supposed that the response of infrared detector is linear and that of designated pixels also remain stable. This method has been implemented especially in military applications $[2,3]$. For this method, a periodic calibration for the system is needed to remove the influence of the drifting of parameters, which increase the complexity and decrease the reliability of the system [4-6]. The second one is scenebased correction, the influence of the drifting of parameters could be reduced by applying this method. But, some preconditions were required to obtain a precise and wide range of adaptive correction, for example, the random scene [7-9]. The scene-based correction is rarely used in hardware systems because of the complexity and the limitations of environmental adaptability of the algorithm itself.

In addition, noise removal is the other critical part of the processing of the infrared images. Algorithms such

*corresponding author; e-mail: xyzeng617@163.com as denoising based on the frequency domain and spatial domain filter, enhancement based on the histogram conversion, and the Retinex algorithm, and so on were widely used to denoise the infrared images in previous researches [10-15]. Different algorithms are suitable for different applicable scenes. In other words, different algorithms have different limitations, no algorithm could be applied to all scenes [16-20]. Therefore, the further research for the correction and enhancement of infrared image should focus on these difficult problems:

1. Present new correction methods and ideas by taking the advantage of richer theoretical and mathematical tools.

2. Build a better theoretical model that contains more related parameters.

3. Faster processing speed.

4. Less bad effects, for example ghost image.

5. Less capacity for the computation and storage.

To solve the above difficult problems, especially the (1) and (4), an algorithm that combined convolutional neural network with double-Gaussian filter that was abbreviated as DGCNN was proposed in this paper. This method produced superior restoration quality of the images and achieved cleaner images with less ghost effect. The contributions of this paper were included as follows:

1. A convolutional neural network combined with double-gaussian filter for noise removal and nonuniformity correction for the infrared images was proposed. 
2. A group of empirical parameters of DGCNN was created to obtain cleaner infrared image with less ghost and noise.

3. The values of roughness and nonuniformity of the infrared images based on different algorithms were calculated to reveal the advantages of DGCNN.

The remainder of this paper is as follows. Section 2 briefly introduces the related theory of DGCNN. Experiments in Sect. 3 compare the results of different algorithms. Section 4 concludes.

\section{Related algorithm theory}

\subsection{Traditional convolutional neural network}

The commonly used convolutional neural network (CNN) algorithm was proposed by Scribner et al. The architecture of this algorithm contains three layers that are the input layer, hidden layer, and output layer, respectively. There are two important processes when the algorithm is working that calculating the actual value of the output and adjusting the weight parameters of the neurons. The grayscale value of each pixel played as the input values would be calculated completely in the hidden layer, then the actual values would be outputted from the output layer. The weight parameter for each pixel will automatically determine and adjust the deviation between the actual output value and the desired output value using valid decision conditions and learning methods. A widely used mathematical module of convolutional neural network algorithm for the uniformity correction of infrared images was expressed as [14]:

$$
Y_{i, j}(k)=G_{i, j}(k) X_{i, j}(k)+O_{i, j}(k),
$$

where $k$ is the sequenced frame number of images, $Y_{i, j}(k)$ and $X_{i, j}(k)$ are corrected and uncorrected responses of $(i, j)$ pixel in the array, respectively. $G_{i, j}(k)$ is the gain correction coefficient of grayscale value of the pixel, $O_{i, j}(k)$ is the offset correction coefficient of grayscale value of the pixel. Besides, a correction of the coefficient $G_{i, j}(k)$ and $O_{i, j}(k)$ based on the bias between the actual value of output and the value of expect output is needed. While, the bias could be expressed as an error function $E_{i, j}(k)$ :

$$
E_{i, j}(k)=\left[Y_{i, j}(k)-f_{i, j}(k)\right]^{2},
$$

where

$$
\begin{aligned}
& f_{i, j}(k)=\frac{1}{4}\left[X_{i-1, j}(k)+X_{i, j-1}(k)+X_{i+1, j}(k)\right. \\
& \left.\quad+X_{i, j+1}(k)\right]
\end{aligned}
$$

is the value of expected output, which is an averaged value of the grayscales of the cross neighborhood pixels of the $(i, j)$ pixel. Moreover, $f_{i, j}(k)$ would be carried back to the hidden layer to compare with the corrected value of output $\left(Y_{i, j}(k)\right)$ by calculating the error function. When $E_{i, j}(k)>\varepsilon$, the correction coefficients $G_{i, j}(k)$ and $O_{i, j}(k)$ would be corrected, where $\varepsilon$ is defined as a suitable threshold. According to Eq. (2), the partial derivative of $E_{i, j}(k)$ with respect to $G_{i, j}(k)$ and $O_{i, j}(k)$ could be respectively written as

$$
\left\{\begin{array}{l}
E_{i, j}^{G}(k)=\frac{\partial E}{\partial G}=2 X_{i, j}(k)\left[Y_{i, j}(k)-f_{i, j}(k)\right] \\
E_{i, j}^{O}(k)=\frac{\partial E}{\partial O}=2\left[Y_{i, j}(k)-f_{i, j}(k)\right]
\end{array}\right.
$$

Then, the $G_{i, j}(k)$ and $O_{i, j}(k)$ could be calculated as

$$
\left\{\begin{array}{l}
G_{i, j}(k+1)=G_{i, j}(k)-2 \alpha X_{i, j}(k)\left[Y_{i, j}(k)-f_{i, j}(k)\right] \\
O_{i, j}(k+1)=O_{i, j}(k)-2 \alpha\left[Y_{i, j}(k)-f_{i, j}(k)\right]
\end{array}\right.
$$

where $\alpha$ is the iteration step-size or learning rate. It should be known that

$$
\begin{aligned}
& f_{i, j}(k)=\frac{1}{4}\left[X_{i-1, j}(k)+X_{i, j-1}(k)+X_{i+1, j}(k)\right. \\
& \left.\quad+X_{i, j+1}(k)\right]
\end{aligned}
$$

is equal to a mean filter based on the four-neighborhood. It is the traditional neural network with an open-loop structure because the $X_{i, j}(k)$ is an uncorrected output. However, the complicated nonuniformity and noise of the infrared image could not be corrected effectively by using the traditional convolutional neural network with an open-loop structure.

\subsection{Convolutional neural network based on double-Gaussian filter}

In order to correct a complicated nonuniformity and noise of the infrared image, the method incorporated the double-Gaussian filter algorithm into the traditional neural network algorithm was performed to adjust the weight parameters of gain correction coefficient $G_{i, j}(k)$ and the offset correction coefficient $O_{i, j}(k)$. The schematic diagram of convolutional neural network is presented in Fig. 1.

As the schematic diagram shows that the critical part of this structure of neural networks is to define a suitable and reliable error function to correct the gain correction coefficient $G_{i, j}(k)$ and offset correction coefficient $O_{i, j}(k)$. Now, we select a pixel area whose size is $[a, b]$, and define the expected output $f_{i, j}(k)$ as [21-23]:

$$
f_{i, j}(k)=\frac{\sum_{a, b} Y_{i, j}(k) W_{i j}}{\sum_{a, b} W_{i j}} .
$$

The weight parameter $W_{i j}$ is defined as:

$$
W_{i j}=H_{i, j} \exp \left(-\frac{\left(Y_{j}-Y_{i}\right)^{2}}{\sigma_{s}^{2}}\right) \exp \left(-\frac{\left(I_{j}-I_{i}\right)^{2}}{\sigma_{r}^{2}}\right)
$$

where $H_{i, j}$ is a normalized constant, $i$ and $j$ are the indices of pixels, $Y$ and $I$ are the spatial and the grayscale values of pixels, $\sigma_{s}, \sigma_{r}$ are the random variable variance of the spatial and grayscale values of the pixels, respectively. $\sigma_{s}$ is regarded as an integer value, and the value of $\sigma_{r}$ ranges from 0 to 1 . Substitute Eqs. (5) and (6) into Eq. (4), the modified gain correction coefficient $G_{i, j}(k)$ and offset correction coefficient $O_{i, j}(k)$ could be expressed as 


$$
\left\{\begin{array}{l}
G_{i, j}(k+1)=G_{i, j}(k)-2 \alpha X_{i, j}(k)\left[Y_{i, j}(k)-\frac{\sum_{a, b} Y_{i, j}(k) H_{i, j} \exp \left(-\frac{\left(Y_{j}-Y_{i}\right)^{2}}{\sigma_{s}^{2}}\right) \exp \left(-\frac{\left(I_{j}-I_{i}\right)^{2}}{\sigma_{r}^{2}}\right)}{\sum_{a, b} H_{i, j} \exp \left(-\frac{\left(Y_{j}-Y_{i}\right)^{2}}{\sigma_{s}^{2}}\right) \exp \left(-\frac{\left(I_{j}-I_{i}\right)^{2}}{\sigma_{r}^{2}}\right)}\right] \\
O_{i, j}(k+1)=O_{i, j}(k)-2 \alpha\left[Y_{i, j}(k)-\frac{\sum_{a, b} Y_{i, j}(k) H_{i, j} \exp \left(-\frac{\left(Y_{j}-Y_{i}\right)^{2}}{\sigma_{s}^{2}}\right) \exp \left(-\frac{\left(I_{j}-I_{i}\right)^{2}}{\sigma_{r}^{2}}\right)}{\sum_{a, b} H_{i, j} \exp \left(-\frac{\left(Y_{j}-Y_{i}\right)^{2}}{\sigma_{s}^{2}}\right) \exp \left(-\frac{\left(I_{j}-I_{i}\right)^{2}}{\sigma_{r}^{2}}\right)}\right]
\end{array}\right.
$$

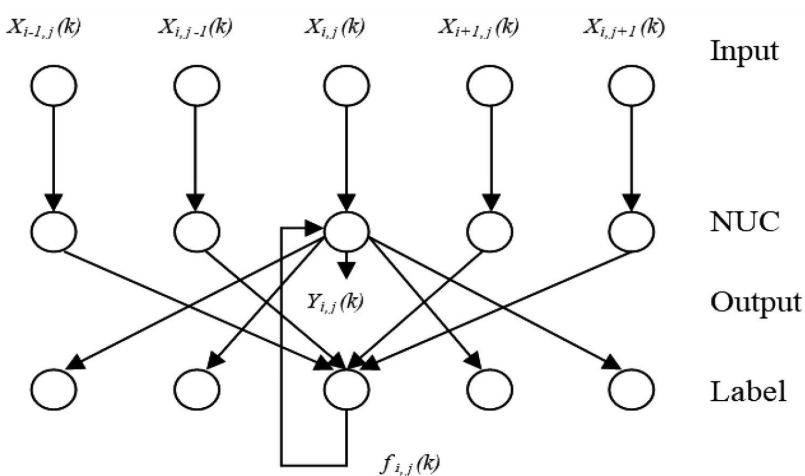

Fig. 1. The schematic diagram of convolutional neural network.

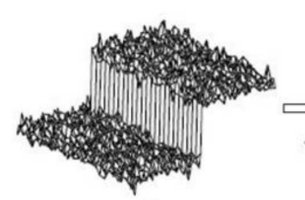

(a)

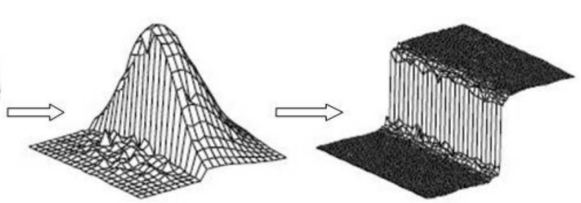

(b) (c)
Fig. 2. The schematic diagram of double-Gaussian filter.

Actually, the weight parameter $W_{i j}$ combined domain and range filtering denoted as double-Gaussian filtering in this paper. It replaces the pixel value at $i$ with an average of similar and nearby pixel values. In a small smooth neighborhood region, pixel values are similar to each other, and the double-Gaussian filter acts essentially as a common domain filter that averaged away the small, weakly correlated differences between pixels caused by noise. As presented in Fig. 2a, there is a sharp boundary between a dark and a bright region. When the doubleGaussian filter is centered on a pixel on the bright side of the boundary, the filter assumes values that close to one for pixels on the same side, and values close to zero for pixels on the dark side. As shown in Fig. 2b for a filter support centered two pixels to the right of the step in Fig. 2a. The weight parameter $W_{i j}$ ensured the weights for all the pixels add up to one. As a result, the double-Gaussian filter essentially ignored the dark pixels, and replaced the bright pixel at the center by the averaged bright pixels in its vicinity. Conversely, when the double-Gaussian filter centered on a dark pixel, the bright pixels are essentially ignored instead. Therefore,

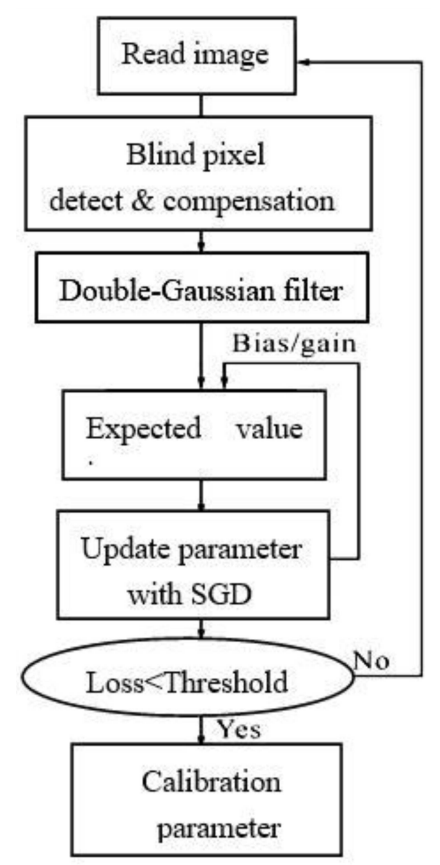

Fig. 3. Software diagram of DGCNN.

Empirical parameters of DGCNN.

TABLE I

\begin{tabular}{c|c|c|c|c|c}
\hline \hline Parameter & $w$ & $\sigma_{s}$ & $\sigma_{r}$ & Iterations & $\begin{array}{c}\text { Optimization } \\
\text { algorithm }\end{array}$ \\
\hline value & 5 & 3 & 0.3 & 50 & SGD
\end{tabular}

as shown in Fig. 2c, good filtering behavior is achieved at the boundaries because of the domain component of the filter, and crisp edges are preserved at the same time benefits from the range component.

Besides, in order to make the calculation of this algorithm that combined double-Gaussian filter with convolutional neural network become much more efficient, the stochastic gradient descent (SGD) method was used. SGD is an online learning method that does not need to store data in advance, while put the input data into the model for training directly to reduce the storage requirements and increase learning efficiency.

Therefore, the method used to denoise and correct the infrared images named DGCNN was a convolutional neural network that incorporated with double-Gaussian filter and the SGD. The software diagram of this method was presented in Fig. 3. 
Besides, the parameters of DGCNN were listed in Table I. These parameters were selected empirically according to a series of experiments carried out for the best results of correction and denoising. The half width of the kernel is assigned as $w=5$, and the standard variance of the double-Gaussian filter is sigma $=[3,0.3]$, respectively.

\section{Results and discussion}

300 infrared images with the nonuniformity and noise that captured from different fields of view were used as the experiment data. As presented in Fig. 4, the first infrared image of the data set was Fig. 4a, the 100th of that was Fig. 4b, the 200th of that was Fig. 4c, and the 300 th of that was Fig. 4d. Obviously, these four images with different degrees of nonuniformity and noise, and all the images were captured with various distance of focus from different fields of view.

In order to illustrate the advantage of DGCNN, which was compared with other algorithms used for the correction and denoising of infrared thermal images, such as back propagation neural networks correction (BPCNN), constant-statistics correction (CSC), temporal high pass filter correction (THPFC), neural networks correction based on median filter (MEDCNN), neural networks correction based on edge detection (EDCNN), and an improved constant-statistics correction (ICSC). As known, BPCNN and CSC are very practical algorithms to adjust the nonuniformity of the infrared image, and many algorithms had been deduced from the two algorithms and become the one that correct and enhance the infrared images much more efficient. It should be noted that all the algorithms mentioned above belonged to the kind of scene-based correction. Furthermore, all algorithms performed in this paper were carried out by using MATLAB 2016, Natick, Massachusetts, USA.

As a result, the various effects of these algorithms on the 300th image of experiment data were presented in Fig. 5. In this experiment, the traditional CNN was changed into a closed-loop CNN by defining the

$$
\begin{aligned}
& f_{i, j}(k)=\frac{1}{4}\left[Y_{i-1, j}(k)+Y_{i, j-1}(k)+Y_{i+1, j}(k)\right. \\
& \left.\quad+Y_{i, j+1}(k)\right]
\end{aligned}
$$

as expected output value that would be feedback to the NUC layer to modify the parameters of error function. As shown in Fig. 5a, it is the raw image with noise and nonuniformity, and the corrected result of CNN was shown in Fig. 5b which smoother than the raw one.

CSC is a classical algorithm to correct the nonuniformity of infrared thermal images, and the core concept of CSC is supposed that the mean and variance of pixels response are consistent within a long period of time, and the randomness of the scene is variable. As shown in Fig. 5c, there are effects of ghost images in the correction result of CSC, because the infrared detector array suffered a back scene of weak randomness. While, ICSC

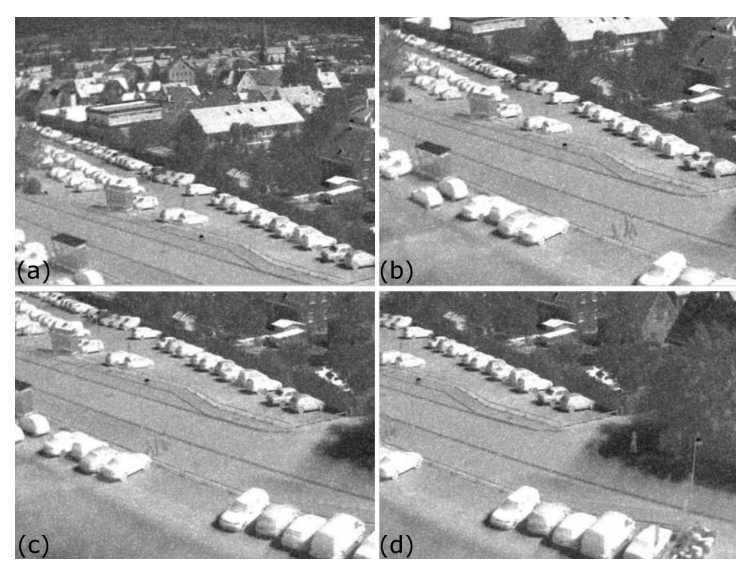

Fig. 4. Four images with different degrees of nonuniformity and noise that captured with various distance of focus from different fields of views.
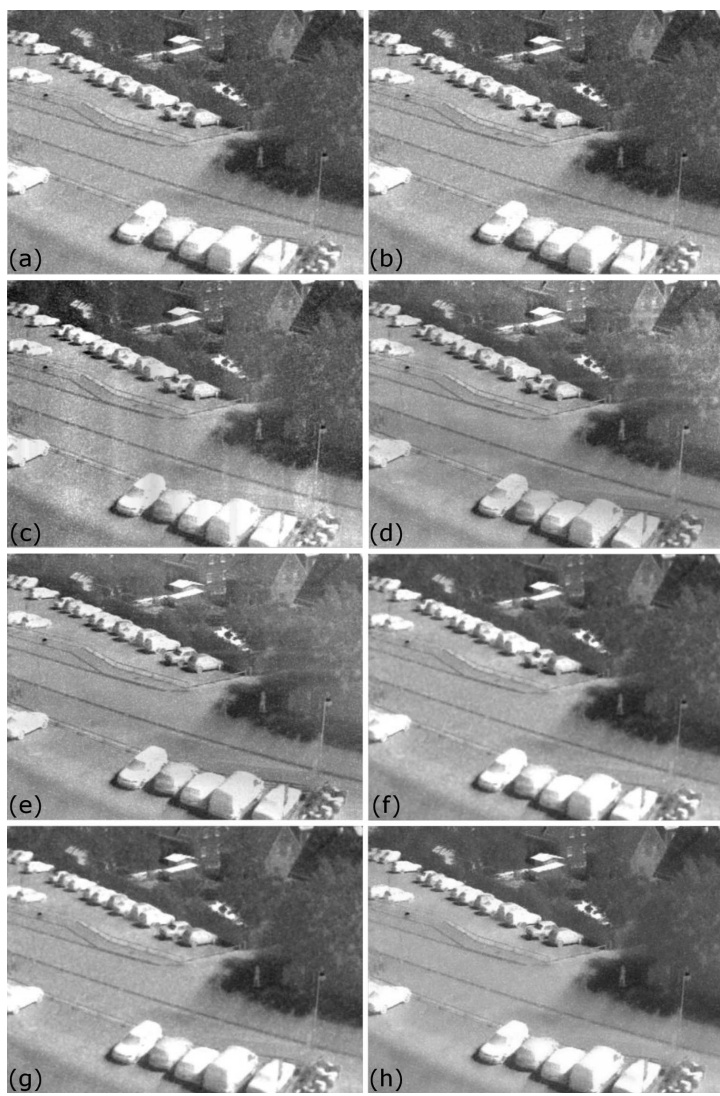

Fig. 5. Correction results of different algorithms. (a) Raw, (b) CNN, (c) CSC, (d) ICSC, (e) THPFC, (f) EDCNN, (g) MEDCNN, (h) DGCNN.

is an improved CSC algorithm in this paper by changing the expectation and variance of the response of detector into the mean value and variance of the pixels. As Fig. $5 \mathrm{~d}$ shows, the result of ICSC is smoother and with better contrast than CSC because the effects of ghost had been reduced by strengthened randomness of the back scene. 
THPFC is a statistical filter based on time domain, supposing the nonuniformity noise of images is mixed with the clutter of background. The images processed by THPFC are separated into two parts, whose object in the scene is the part of low frequency and nonuniformity noise is the part of high frequency. Only the part of high frequency is used to correct the nonuniformity, which is conductive to correct the images in a proper way and avoid the effects of ghost images. As Fig. 5e depicts, the correction result of THPFC is better than that of CNN and CSC, which is smoother than CNN while sharper than CSC. However, EDCNN and MEDCNN are two algorithms that were derived from CNN. Actually, MEDCNN combined a mean filter with the CNN.

In this paper, a $3 \times 3$ array as a kernel of the mean filter was assigned to sort the sampling data in the neighborhood and obtain the median value to determine the central gray pixel value. The central gray pixel value would be used as the expected output value feedback to the NUC layer of the CNN to modify the parameters of error function. While, EDCNN is a combination of the edge detection and CNN by adding the sobel operator into the CNN algorithm. As known, mean filter is a good choice for the smoothness of the noise while sobel operator is commonly used to distinguish the edges in picture. As depicted in Fig. 5f and Fig. 5g, the results of the two algorithms of EDCNN and MEDCNN are smoother than other results shown in Fig. 5a-e. Besides, there are more details remaining in Fig. $5 f$ than in Fig. $5 \mathrm{~g}$ because the sobel operator kept the edges in picture from being filtered. Furthermore, DGCNN proposed in this paper is the algorithm that combined the double-Gaussian filter with the convolutional neural networks to correct the nonuniformity and remove the noise of infrared thermal images. Actually, the error function of traditional CNN was rewritten by adding weight parameters into the gain correction coefficient $G_{i, j}(k)$ and the offset correction coefficient $O_{i, j}(k)$, as presented in Eq. (7). Thus, the advantages of DGCNN can be concluded as the values of the spatial pixels and grayscale could be calculated simultaneously by iterating in the error function and adjusting the weight parameters, which is beneficial to decrease noise and remain details of the infrared thermal images. As presented in Fig. 5h, the correction result of DGCNN is better than all the algorithms mentioned above, which is smoother and sharper.

Besides, Fig. 6 is a clean infrared thermal image without noise and nonuniformity. It is seen that Fig. 5h is very close to the clean images, and that the contrast of Fig. $5 \mathrm{~h}$ could be changed to get a same result like Fig. 6 . According to the depiction above, the DGCNN is a better algorithm for the correction and denoising of the infrared thermal image. However, it is not clear for us to distinguish the slight difference among these images by eyes. To make a clearer contrast, the values of roughness and nonunformity of all the 300 corrected images were calculated, respectively. The roughness was regarded as a representation of smoothness of the infrared thermal

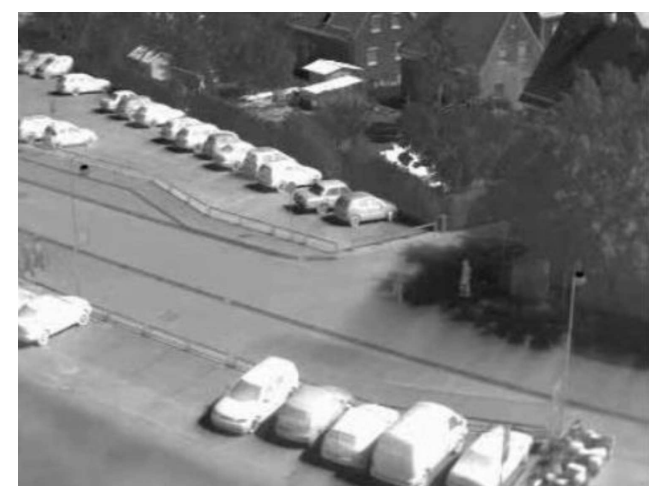

Fig. 6. A clean infrared thermal image without noise and nonuniformity.

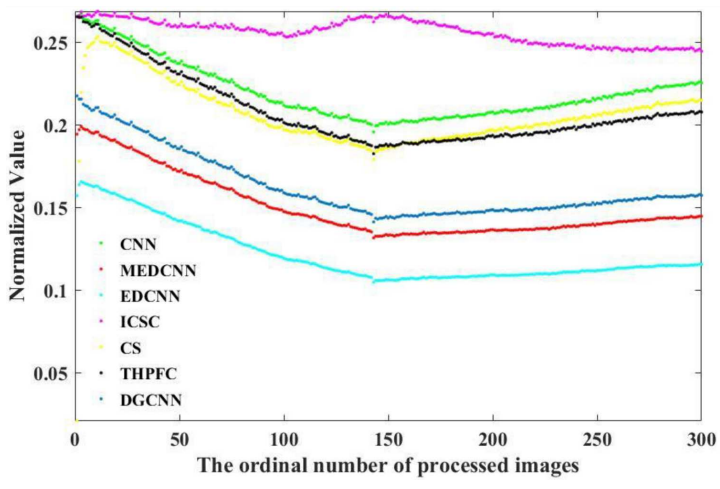

Fig. 7. Roughness of the infrared thermal image processed by all algorithms.

images, and a smaller value of roughness corresponds to a smoother image. The value of roughness for each of the 300 infrared thermal images was calculated and normalized to a range from 0 to 1 . As Fig. 7 presented, the sequential number of these images from 1 to 300 was horizontal coordinate while the normalized value of roughness as vertical coordinate. It was seen that roughness of the 300 infrared thermal images processed by different algorithms mentioned above can be ordered from lower to higher order as: EDCNN $<$ MEDCNN $<$ DGCNN $<$ $\mathrm{CSC}<\mathrm{THPFC}<\mathrm{CNN}<\mathrm{ICSC}$.

However, the value of roughness is not the only one factor to define the quality of these infrared thermal images. Besides, the nonunformity is the other essential factor to affect the quality of infrared thermal images. It is known that the value of nonunformity of infrared thermal image was defined as the ratio of standard variance and mean value of responses of all the effective pixels that can be written as [24]:

$$
\left\{\begin{array}{l}
\mathrm{NUV}=\frac{1}{V_{\mathrm{avg}}} \sqrt{\frac{1}{M N-(d+h)} \sum_{i=1}^{M} \sum_{j=1}^{N}\left(V_{i j}-V_{\mathrm{avg}}\right)^{2}}, \\
V_{\mathrm{avg}}=\frac{1}{M N-(d+h)} \sum_{i=1}^{M} \sum_{j=1}^{N} V_{i j},
\end{array}\right.
$$

where $M$ and $N$ are the number of pixels in the row and column of the infrared detector array, respectively. $V_{i j}$ is the response of the pixel at the position of $(i, j)$ 


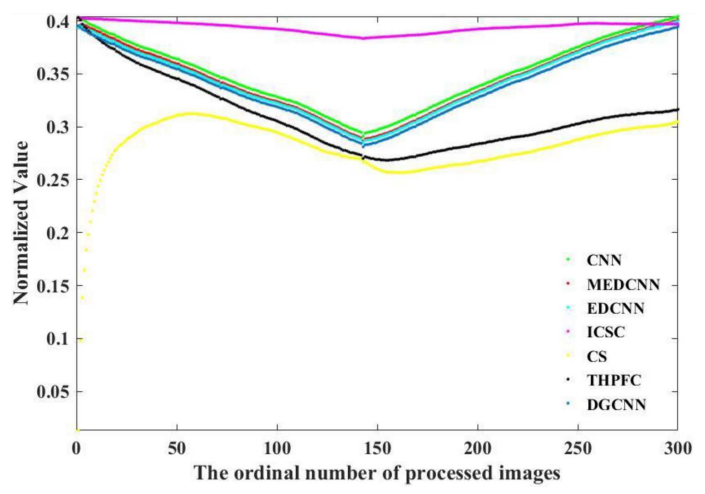

Fig. 8. Nonunformity value of the infrared thermal image processed by all algorithms.

while $V_{\text {avg }}$ is the mean value of the responses of effective pixels in the infrared detector array. Besides, $d$ and $h$ are the number of dead pixels and over hot pixels, respectively. As Fig. 8 depicted, the values of nonuniformity calculated out by different algorithms mentioned above could be ordered from lower to higher order as: CS < THPFC $<$ DGCNN $<$ EDCNN $<$ MEDCNN $<$ CNN $<$ ICSC.

As described above, the value of roughness calculated based on DGCNN is higher than EDCNN and MEDCNN while lower than that of others, which means DGCNN is neither the best nor the worst choice from the point of roughness. The value of nonuniformity obtained on the base of DGCNN is lower than EDCNN and MEDCNN, which means DGCNN is the better choice than that two algorithms from the point of nonuniformity. In summary, DGCNN is neither the best nor the worst one from the point of roughness and nouniformity while it is the best one from a practical point view. Therefore, the DGCNN is a potential method for the better correction and denoising of infrared thermal images.

\section{Conclusion}

A potential method for the nonuniformity correction and noise removal of the infrared thermal images that combined convolutional neural networks with a doubleGaussian filter was proposed, which was expressed as DGCNN. To demonstrate the advantages of this method, 300 infrared thermal images with different degrees of nonuniformity captured from various focal distances and fields of view were analyzed. Particularly, the value of roughness and nonuniformity for each infrared thermal image were calculated based on DGCNN. Besides, the results of that were compared in the form of a chart line with other commonly used algorithms, such as BPCNN, CSC, THPFC, MEDCNN, EDCNN, and ICSC. As a result, DGCNN was neither the best nor the worst one from the point of roughness and nouniformity while it was the best one from a comprehensive performance of view. Therefore, the DGCNN may be a potential method for the nonuniformity correction and denoising of the infrared thermal image.

\section{Acknowledgments}

This work was supported by National Key Research and Development Plan "Earth Observation and Navigation" Key Special Project (2017YFB0503102).

\section{References}

[1] J.M. Mooney, F.D. Shepherd, Infrared Phys. Technol. 37, 595 (1996).

[2] J.G. Harris, Y.M. Chiang, IEEE Trans. Image Process. 8, 1148 (1999).

[3] M. Schulz, L. Caldwell, Infrared Phys. Technol. 36, 925 (1995).

[4] W. Zhao, C. Zhang, J. Opt. Soc. Am. A 25, 1668 (2008).

[5] Y. Liu, H. Zhu, Y. Zhao, Appl. Opt. 48, 2364 (2009).

[6] E. Vera, P. Meza, S. Torres, Opt. Lett. 36, 172 (2011).

[7] N. Liu, J. Xie, Infrared Phys. Technol. 69, 198 (2015).

[8] B. Narayanan, R.C. Hardie, R.A. Muse, Appl. Opt. 44, $3482(2005)$.

[9] N. Liu, X. Chen, Infrared Phys. Technol. 77, 405 (2016).

[10] Y. Li, Y. Zhang, A. Geng, L. Cao, J. Chen, Opt. Laser Technol. 83, 99 (2016).

[11] C. Zuo, Q. Chen, G. Gu, W. Qian, Opt. Rev. 18, 197 (2011).

[12] A. Rossi, M. Diani, G. Corsini, Opt. Eng. 49, 057013 (2010).

[13] S.C. Cain, M.M. Hayat, E.E. Armstrong, IEEE Trans. Image Process. 10, 1860 (2001).

[14] L. Rui, Y. Yin-Tang, Z. Duan, L. Yue-Jin, Appl. Opt. 47, 4331 (2008).

[15] J.H. Kim, S.W. Jung, C.K. Noh, S.J. Ko, Opt. Eng. 50, 077002 (2011).

[16] R. Lai, Y.T. Yang, B.J. Wang, H.X. Zhou, Opt. Commun. 283, 4283 (2010).

[17] Bai Jun-Qi, Chen Qian, Wang Xian-Ya, Qian WeiXian, Infrared Laser Eng. 39, 777 (2010) (in Chinese).

[18] K. Liang, Y. Ma, Y. Xie, B. Zhou, R. Wang, Infrared Phys. Technol. 55, 309 (2012).

[19] Y. Li, Y. Zhang, A. Geng, L. Cao, J. Chen, Opt. Laser Technol. 83, 99 (2016).

[20] Shih-Chih Huang, Chien-HuiYeh, Eng. Appl. Artific. Intell. 1487-1492, 26 (2013).

[21] Li Zhaolong, Shen Tongsheng, Lou Shuli, Infrared Phys. Technol. 77, 360 (2016).

[22] Zhang Long, Dong Feng, Fu Yutian, Infrared Technol. 36, 164 (2014).

[23] J.Q. Bai, C.W. Chang, W. Liu, Lect. Notes Electr. Eng. 474, 677 (2018).

[24] S.H. Rong, H.X. Zhou, H.L. Qin, R. Lai, K. Qian, Infrared Phys. Technol. 76, 691 (2016). 